\title{
ANTS 2018 special issue: Editorial
}

\author{
Marco Dorigo ${ }^{1}$ - Mauro Birattari ${ }^{1}$. Christian Blum ${ }^{2}$. Anders L. Christensen ${ }^{3}$. \\ Andreagiovanni Reina ${ }^{4} \cdot$ Vito Trianni ${ }^{5}$
}

Published online: 28 October 2019

(c) Springer Science+Business Media, LLC, part of Springer Nature 2019

This special issue of the Swarm Intelligence journal is dedicated to the publication of extended versions of some of the best papers presented at ANTS 2018, Eleventh International Conference on Swarm Intelligence, which took place in Rome, Italy, on October 29-31, 2018.

ANTS is the first and most established conference series dedicated to the dissemination of swarm intelligence research. Its first edition took place in 1998, at the Université Libre de Bruxelles, Brussels, Belgium. Since then, it has been held every other year, and since 2010 (i.e., starting with the seventh edition of the conference), the authors of the full-length papers accepted at the conference were invited to submit an extended version for possible inclusion in a dedicated special issue of the Swarm Intelligence journal.

Ten articles from the 2018 edition of the ANTS conference were accepted for publication in the special issue after at least two rounds of reviews with comments by at least three referees. The papers of which one of the authors is also a guest editor of this special issue have been managed anonymously by one of the other guest editors.

The special issue is organized in two parts. The first part is this double issue, and the second part will be published as the first issue of year 2020 (volume 14, number 1). In the following, each paper is briefly introduced by the guest editor who managed it.

$凶 \quad$ Marco Dorigo

mdorigo@ulb.ac.be

Mauro Birattari

mbiro@ulb.ac.be

Christian Blum

christian.blum@iiia.csic.es

Anders L. Christensen

andc@mmmi.sdu.dk

Andreagiovanni Reina

a.reina@sheffield.ac.uk

Vito Trianni

vito.trianni@istc.cnr.it

1 IRIDIA, Université Libre de Bruxelles, Brussels, Belgium

2 Artificial Intelligence Research Institute, IIIA-CSIC, Barcelona, Catalonia, Spain

3 MMMI, University of Southern Denmark, Odense, Denmark

4 University of Sheffield, Sheffield, UK

5 Institute of Cognitive Sciences and Technologies, CNR, Rome, Italy 
The special issue opens with "The intelligent water drops algorithm: why it cannot be considered a novel algorithm. A brief discussion on the use of metaphors in optimization." In this paper, Christian Leonardo Camacho-Villalón, Marco Dorigo and Thomas Stützle address a disturbing issue: the excessive use of natural metaphors for the development of optimization algorithms, which has become an extremely popular trend in the last fifteen years. In the paper, the authors rigorously analyze the intelligent water drops (IWD) algorithm, which is one such metaphor-based approach for solving discrete optimization problems. The authors demonstrate that all the main algorithmic components of IWD are simplifications or special cases of ant colony optimization (ACO) and that therefore IWD is simply a particular instantiation of ACO. Furthermore, they show that the natural metaphor that inspired IWDnamely "water drops flowing in rivers removing the soil from the riverbed"-is unnecessary, misleading and based on unconvincing assumptions of river dynamics and soil erosion, lacking a scientific rationale. They also carry out a detailed review of modifications and extensions proposed to IWD since its first publication in 2007. Not surprisingly, they find that the vast majority of the ideas explored in the literature on IWD were already studied many years before in the context of ACO. Finally, the authors provide a highly useful discussion of natural metaphors as a source of inspiration for optimization algorithms, and they propose some criteria to limit their usage to the cases in which the metaphor is indeed useful.

In "Degrees of stochasticity in particle swarm optimization," Elre T. Oldewage, Andries P. Engelbrecht and Christopher W. Cleghorn illustrate-in the context of particle swarm optimization (PSO) - the theoretical and empirical importance of independent, component-wise stochastic scaling values. They show that a PSO algorithm employing scalar stochasticity in the particle update equation is unable to express every point in the search space if the problem dimensionality is sufficiently large in comparison with the swarm size. This theoretical result is emphasized by an empirical experiment in which the authors show that, when using scalar stochasticity, PSO performs significantly worse if the optimum is not in the range of the initial positions. Furthermore, they demonstrate that even when the problem dimensionality allows the scalar PSO variant to reach the optimum, a PSO variant with component-wise stochasticity significantly outperforms the scalar variant. This result is extended by considering different degrees of stochasticity, in which groups of components share the same stochastic scalar. The authors demonstrate, on a wide range of benchmark functions, that PSO variants with a dimensional coupling (including the scalar PSO variant in the most extreme case) perform significantly worse than PSO variants with component-wise stochasticity. Finally, the paper also shows that, contrary to previous results reported in the literature, a PSO variant with component-wise stochasticity is not biased toward the subspace within which it is initialized. The misconception, as convincingly argued by the authors, has arisen in the previous literature due to overzealous normalization when measuring swarm movement, which is avoided in this paper.

In "Collective decision making in dynamic environments," Judhi Prasetyo, Giulia De Masi and Eliseo Ferrante study a binary decision problem is swarm robotics, whereby the quality of the two available alternatives abruptly changes at some point in time. To deal with changing environmental conditions, two different adaptive mechanisms are introduced, which operate at different abstraction levels. The first mechanism alters the individual behavior, introducing a spontaneous option-switching probability that lets individuals evaluate other alternatives than the chosen one. The second mechanism operates at the swarm level, introducing a number of 'stubborn' agents that never change opinion. Both mechanisms succeed in dealing with changing environmental conditions and can be tuned to trade adaptation accuracy with decision speed. 
In "Multi-guide particle swarm optimization for multi-objective optimization. Empirical and stability analysis," Christiaan Scheepers, Andries P. Engelbrecht and Christopher W. Cleghorn present a new multi-objective optimization (MOO) algorithm based on particle swarm optimization (PSO), which they name multi-guide particle swarm optimization (MGPSO). MGPSO is a multi-swarm approach in which each subswarm optimizes one of the objectives. An archive is added to the velocity update equation in order to facilitate convergence to a Pareto front of non-dominated solutions. The authors conduct an extensive empirical analysis of MGPSO, with a special focus on stability aspects. The empirical analysis studies the exploration behavior of MGPSO and compares the performance of MGPSO with that of state-of-the-art multi-objective PSO and evolutionary algorithms. The authors are able to show that MGPSO is highly competitive on a number of benchmark functions. On the theoretical side, the paper provides a stability analysis that focuses on the sufficient and necessary conditions for order-1 and order-2 stability of MGPSO. In that respect, the paper extends existing work by deriving new stability criteria for differing values of the acceleration coefficients used in the velocity update equation.

In "The PageRank algorithm as a method to optimize swarm behavior through local analysis," Mario Coppola, Jian Guo, Eberhard Gill and Guido de Croon propose the use of the Google's PageRank algorithm - a well-known graph centrality measure for ranking the importance of web pages - to evaluate and optimize the global behavior of robot swarms. The authors use the PageRank algorithm to determine how likely it is for a robot to visit local states given a local policy. They then use an evolutionary algorithm to optimize the local policy and maximize the probability that a robot will visit states that contribute to the global goal of the swarm. The computational complexity of the PageRank-based approach depends only on the number of local states and not on the size of a swarm, and it thus has the potential to scale well. The novel approach is assessed on three simulated swarm robotics tasks-consensus agreement, pattern formation and aggregation-and the findings show that the controllers are flexible and robust, and that performance is comparable to globally optimized policies.

In "Coherent collective behaviour emerging from decentralised balancing of social feedback and noise," Ilja Rausch, Andreagiovanni Reina, Pieter Simoens and Yara Khaluf study the link between noise and social feedback, and how this relationship affects group coherence in a decentralized system of embodied agents. The authors use a locust-inspired case study of marching agents modeled as a binary collective decision-making problem, in which the amount of social feedback received by an agent is determined by the number of communication links it has. The minimal number of communication links necessary to attain maximum collective coherence is approximated analytically, and the theoretical results are validated in physics-based simulations. The authors also present experiments in which robots use a decentralized algorithm to adaptively reach coherence for various noise levels by regulating the number of communication links.

In "Toward a theory of collective resource distribution: a study of a dynamic morphogenesis controller," Payam Zahadat and Daniel Nicolas Hofstadler propose a distributed algorithm to select locations to add new modules or remove existing modules of a dynamic physical structure. The algorithm that runs on each module of the physical structure is inspired by plant morphogenesis and controls the allocation of resources for growth. This study investigates the effect of the algorithm parameters on the resulting collective behavior. The predictions of the theoretical analysis are confirmed through the physical implementation of the algorithm on a cyber-physical modular system. Interestingly, the authors identify similarities between their algorithm and other decentralized processes, contributing to a unified view of collective systems. 
Part 2 of the special issue starts with the article "Simulation-only experiments to mimic the effects of the reality gap in the automatic design of robot swarms," by Antoine Ligot and Mauro Birattari. The authors propose a methodology to understand how sensitive an automatic design method is to changes in the testing environment. When offline automatic design is employed to synthesize the control system for (swarms of) robots, the unavoidable difference between simulation model and the real environment usually leads to a drop in performance. This is essentially due to the design method overfitting some features of the simulated environment and therefore showing a lack of generalization to the real world. In the paper, the authors show that such overfitting can be observed also in two simulation models that differ only by some parameters (e.g., the level of noise experienced by the robots). In this way, they demonstrate that it is not necessary to have a difference in kind between training and testing environments (e.g., simulation versus reality) to observe the performance drop typical of the reality-gap problem.

In "Sophisticated collective foraging with minimalist agents: a swarm robotics test," Mohamed S. Talamali, Thomas Bose, Matthew Haire, Xu Xu, James A. R. Marshall and Andreagiovanni Reina investigate the achievement of efficient collective foraging in natural and robotic swarms. The authors explore the effect of swarm size on foraging efficiency and the trade-off between distance to and quality of a foraging site. Although the robots are only equipped with binary pheromone sensors, the authors are able to reproduce classical foraging experiments that were conducted with real ants that sense the concentration of pheromone and follow its gradient. The authors construct an optimal foraging theory model that accounts for distance and quality of resources, as well as overcrowding, and predicts a strategy dependent on swarm size. The strategy is successfully validated in experiments with up to 200 simulated and physical Kilobot robots.

In "A study on force-based collaboration in swarms," Chiara Gabellieri, Marco Tognon, Dario Sanalitro, Lucia Pallottino and Antonio Franchi study the control problem of stabilizing a load cooperatively transported by multiple robots. The authors consider both airborne and ground robots and address the case of the load being transported through elastic links (e.g., ropes, springs). While all the robots transporting the load can exert a force to stabilize the attitude of the load, only a few of them have knowledge of its desired position and orientation. These are considered the leaders of the team, while others are referred to as followers. The authors study the conditions under which the system attains a stable equilibrium, and study its robustness against external forces and random disturbances.

The high quality of the 10 papers contained in this special issue is the result of the collaboration of a large number of people: the authors, who submitted their best work to the journal; the referees, who helped in the selection of the published papers; and finally, the many people at Springer who assisted us in the production phase. We thank them all for their help to make this special issue possible.

Publisher's Note Springer Nature remains neutral with regard to jurisdictional claims in published maps and institutional affiliations. 